\title{
Valoración de cualidades físicas en mujeres adultas antes y después de un programa de ejercicio físico
}

\author{
Women's physical status, before \\ and after an exercise program:
}

•PSS. Lic.Enf. Luz Nayeli Huerta Barrrera.

•.Lic. Enf.Beatriz Ruiz Padilla, ...Dra. Maria Cristina Rodriguez Gutierrez.

\section{Resumen}

Introducción: Las cualidades físicas en la vida cotidiana son la expresión de numerosas funciones corporales, la pérdida progresiva de estas, constituye un problema de salud debido al deterioro del estado funcional de la persona modificando su calidad de vida, es necesario fomentar el buen estado de salud para poder llegar a edades más avanzadas de la vida con un mantenimiento y/o mejoramiento de las cualidades físicas para disfrutar plenamente la vida. Objetivo: Conocer los cambios en las cualidades físicas de mujeres adultas sedentarias evaluándolas comparativamente al inicio y término de un programa de entrenamiento físico. Metodología: Diseño del estudio prospectivo, longitudinal y comparativo, la población de estudio fue de mujeres de 45 a 60 años de edad, sedentarias, la muestra se constituyo a conveniencia con 47 mujeres que terminaron el programa de entrenamiento físico, las cualidades físicas se evaluaron antes y después de un programa de ejercicio físico. Para conocer el comportamiento de la muestra se obtuvieron medidas de tendencia central, dispersión y pruebas de correlación. Resultados: En la primera valoración las cualidades físicas que se encontraban con un mayor nivel de deficiencia fueron la fuerza $(92 \%)$, flexoelasticidad de cadera $(93 \%)$ y flexoelasticidad de mano $(66 \%)$, al realizar la segunda valoración las cualidades físicas que alcanzando en promedio un incremento significativo estadísticamente fueron la fuerza con 1.2 Kg, la velocidad de reacción visual con 1.1 mseg, la flexoelasticidad de tronco con

- Pasante de la Licenciatura de Enfermeria ENEO.

- Profesor de carrera asociado A EnEO. Coordinadora de la Especialidad de Enfermeria de la

Cultura Fisica y El deporte.

...Directora de Medicina del Deporte unam.

Correspondencia: uriel_797@hotmail.com • beatrizrp@servidor.unam.mx

RECIBIDO 24-07-2010

ENVIADO31-07-2010

ACEPTADO 2-08 2010 


\section{Enfermería Universitaria}

Resumen

3.6 cm y la flexoelasticidad de mano con 0.9 cm. Discusión: Coincidimos con Sánchez Pinilla en lo referente a que la capacidad para mejorar las cualidades físicas dependerá de la cantidad de trabajo y tipo de ejercicio físico especifico para cada una de ellas, lo cual pudo haber contribuido a una mejora más significativa de las cualidades físicas. Conclusiones: De acuerdo con los resultados el presente estudio es una herramienta que puede ser empleada para analizar los efectos del ejercicio físico sobre las cualidades físicas.

\section{AbstRACT}

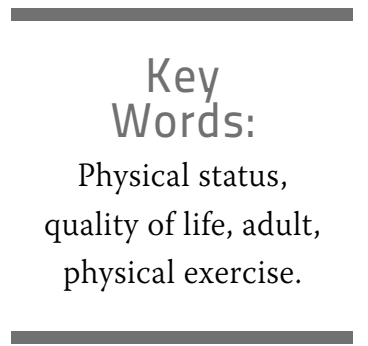

Introduction: Physical status is the expression of several body functions, and its progressive impairment represents a health problem; therefore, it is necessary to promote healthy habits in people so that they can enjoy a high quality of life for as long as possible. Objective: To address the physical status changes in sedentary women before and after their participation in an exercise program. Methodology: Prospective study, addressing the physical status of 47, 45-60 year-old, sedentary women before and after an exercise program. The SPSS v16 was used to obtain central tendency, dispersion, and correlation data. Results: In the first assessment, strength, hip flex-elasticity, and hand flex-elasticity were found to be the most impaired. In the second assessment, the physical capacities which showed statistically significant average increments were: strength (1.2 kg), visual reaction velocity $(1.1 \mathrm{msec})$, trunk flex-elasticity $(3.6 \mathrm{~cm})$, and hand flex-elasticity $(0.9 \mathrm{~cm})$. Discussion: We agree with Sanchez Pinilla that the capacity to improve physical performance will depend on the quantity and type of exercise involved. Conclusion: Considering the results, this study can be a reference to help analyze the effects of exercise on the physical status.

\section{INTRODUCCION}

Las cualidades físicas constituyen la expresión de numerosas funciones corporales que permiten la realización de las diferentes actividades en la vida cotidiana, como: alimentarse, dormir, desplazarse, vestirse o asearse, así como las tareas laborales, profesionales y caseras. ${ }^{1,2,3}$

Según Carbonell Durante el trascurso de la vida se produce una involución en las cualidades físicas, la pérdida progresiva de la fuerza, flexoelasticidad, velocidad de reacción y equilibrio, origina un deterioro del estado funcional, lo que impide el disfrute pleno de la exis- tencia de la persona, alterando su calidad de vida., 4

Estos hechos condicionan graves problemas de salud pública por la aparición de enfermedades crónico degenerativas con el consiguiente costo económico para su tratamiento, repercutiendo en la población mexicana, estimando que el número de la población adulta se acentuara debido a la inversión de la pirámide poblacional que para el año 2015 será de 11, 369,149 de adultos mayores. ${ }^{5,6,7}$

Para enfrentar este problema es necesario mantener y/o mejorar las cualidades físicas, propiciar en el cuerpo un buen estado de salud, obtener la disponibilidad de energía necesaria para apreciar y disfrutar mejor los placeres de la vida, llevar a cabo diariamente tareas con vigor, esto significa hacer más cosas y de más calidad, en todas las esferas de la vida: psicológico, físico y social, para llegar a edades mayores con mejor disposición al trabajo y menor dependencia física. ${ }^{8}, 9,10,11$

Las cualidades físicas se clasifican en básicas, complementarias y derivadas: las cualidades básicas son flexoelasticidad, fuerza y velocidad de reacción, estas participan de manera indispensable en 
la mayoría de las actividades; las cualidades complementarias son, equilibrio, coordinación ubicación espacial, que deben estar presentes en cualquier actividad para un óptimo desempeño pero no son indispensables para la vida y las cualidades derivadas son la conjunción de las cualidades básicas o complementarias. ${ }^{8,9}$

La flexoelasticidad es la capacidad para mover una articulación a través de su rango total, esta cualidad física es la primera que por lo general disminuye debido al deterioro progresivo de los encadenamientos cruzados del colágeno (artrosis y anquilosis articular), dando como resultado una perdida de extensibilidad y un aumento de la rigidez. ${ }^{4,10}$

Para mejorar la flexoelasticidad se debe trabajar con ejercicios simples que obliguen a las articulaciones alcanzar su rango completo de movimiento, utilizándose el estiramiento que consiste en la elongación forzada de un grupo muscular, mediante movimiento con rebote $o$ mantenimiento estático y sostenido hasta el límite en que se hace molesto. $^{8,10,12}$

Otra cualidad física que con mayor frecuencia resulta deficiente produciendo dependencia para determinadas funciones vitales es la "fuerza" entendiéndose como la aptitud para vencer una resistencia estática o dinámica exterior siendo una de las cualidades que forma parte de cualquier manifestación del movimiento controlado de manera voluntaria, por simple que éste sea, esto significa que se aplica fuerza al hablar, al escribir, sentarnos, levantarnos, caminar, correr, saltar etc. Algunos estudios han demostrado una asociación entre el déficit de fuerza en miembros pélvicos y las caídas. ${ }^{10,13}$

Esta cualidad se mejora al entrenar la fuerza con el uso de cargas pesadas y con series de pocas repeticiones, lo que provoca aumento de masa muscular. ${ }^{10}$

Cabe mencionar que los almacenes intramusculares de ATP y fosfocreatina, aumentan con el entrenamiento de la fuerza, al igual que los de glucógeno, sin que se produzcan cambios en la glucosa en sangre por lo cual incrementa la capacidad de utilización de energía anaeróbica. ${ }^{10,12}$

De igual manera existe una discreta mejora en el sistema transportador de oxigeno y su consumo (VO2 máx.), aunque no tanto como en el entrenamiento de resistencia aeróbica. ${ }^{10}$

El equilibrio es la capacidad que tiene el organismo para conservar el centro de gravedad, es una cualidad física sensorio motriz constituida por la propiocepción, la visión, el sentido vestibular, la función muscular y el tiempo de reacción; los trastornos del mismo son factores que predisponen a la inestabilidad y caídas provocando lesiones que llevan a la incapacidad y están asociados principalmente con el incremento de la edad manifestándose en un bajo rendimiento de las tareas tales como estar de pie, inclinarse, subir escaleras y caminar. $^{4,13}$

Mora Risco, clasifica el equilibrio dentro de la coordinación general, por lo que esta cualidad se entrena al realizar variaciones de la postura, la dinámica del movimiento (ejecución más rápida o más lenta), la estructura espacial del gesto (la estructura del terreno y sus dimensiones), así como ejercicios en forma estática y dinámica en el suelo o sobre bases elevadas. ${ }^{12}$

La velocidad de reacción se refiere a la capacidad del sistema nervioso para recibir un estimulo, identificarlo, decidir y si fuera preciso enviar una indicación al músculos para responder; esta se trabaja con esfuerzos de la misma intensidad y de duración muy corta, por debajo de los 15 segundos, es por ello que la adecuada velocidad de reacción disminuye el riesgo de sufrir accidentes, debido a que se adquiere una percepción más amplia de los estímulos visuales, auditivos y táctiles, $8,9,12$

Una ventaja de las cualidades físicas, es que al ser susceptibles de entrenamiento permiten la posibilidad de su mejora o mantenimiento. ${ }^{10}$

Recordemos que el entrenamiento se refiere a la adquisición de conocimientos, habilidades y capacidades resultado de la enseñanza de habilidades, lo mismo sucede con el entrenamiento físico el cual nos presenta una serie planteada de ejercicios físicos que desarrolla habilidades específicas con la intención de conseguir el máximo potencial en un momento determinado, logrando un aprendizaje al adquirir nuevas habilidades, destrezas, conocimientos y conductas, las cuales al estar en continua formación aumentan y adecuan el conocimiento del ejercicio físico. ${ }^{14,15}$

Para la disciplina de Enfermería resulta importante el valorar las cualidades físicas o aptitud física general antes de implementar un programa de ejercicio físico, es indispensable dosificar adecuadamente la carga de trabajo, para que la prescripción del ejercicio físico sea similar a la prescripción de los 
medicamentos se requiera ser pertinente a cada caso para evitar efectos no deseados.

Además de mantener y/o mejorar las cualidades físicas, se asume que el ser humano ha sido diseñado para el movimiento, el cual es indispensable para satisfacer sus necesidades, como sinónimo de salud y calidad de vida.

La participación de enfermería es necesaria para llevar a cabo la valoración de las cualidades físicas, así como el acompañamiento integral durante las sesiones de ejercicio físico y la supervisión de la utilización adecuada de la carga de trabajo.

Siendo importante el presente estudio para corroborar como existe una mejora de las cualidades físicas con el ejercicio físico las cuales mejoran la calidad de vida de las personas.

\section{OBJETIVO}

Conocer los cambios en las cualidades físicas de mujeres adultas sedentarias evaluándolas comparativamente al inicio y término de un programa de entrenamiento físico.

\section{METODOLOGIA}

El presente trabajo es prospectivo, longitudinal y comparativo, la población de estudio fue de mujeres de 45 a 60 años de edad, sedentarias, la muestra se constituyo a conveniencia con 47 mujeres que terminaron el programa de entrenamiento físico. Se excluyeron a las mujeres que habían realizado ejercicio físico organizado durante el último año o presentaran alguna patología que contraindicaran el ejercicio físico. Se eliminaron a las mujeres que no asistieron en forma regular al entrenamiento físico.
Previo consentimiento informado, se evaluó pre y pos ejercicio algunas características antropométricas como peso, talla Índice de Masa Corporal (IMC), porcentaje de grasa. Las cualidades físicas valoradas fueron: Fuerza, flexoelasticidad, velocidad de reacción y equilibrio.

Para medir la fuerza isométrica a máximo esfuerzo de bíceps, cuadriceps, extensores y flexores de tronco se utilizo el dinamómetro universal (Jamar, Back Leg y Chest, made in England). Para la fuerza de mano se utilizo el dinamómetro marca (Harpenden, made in England).

El procedimiento general implicó colocar a la persona de manera que las articulaciones a explorar (rodilla, codo, mano y tronco) se encontraran en su posición intermedia en cuanto a longitud del músculo y perpendicular al segmento óseo.

La longitud del bíceps se midió del cóndilo a la línea media en contracción; el cuadriceps se midió del disco tibial a la línea media del cable del dinamómetro colocado en el tobillo; los extensores y flexores de tronco, en posición ortostática y con flexión de tronco anterior y posterior (figura1).

\section{Figura 1. Prueba de extensores de tronco en dinamómetro universal.}

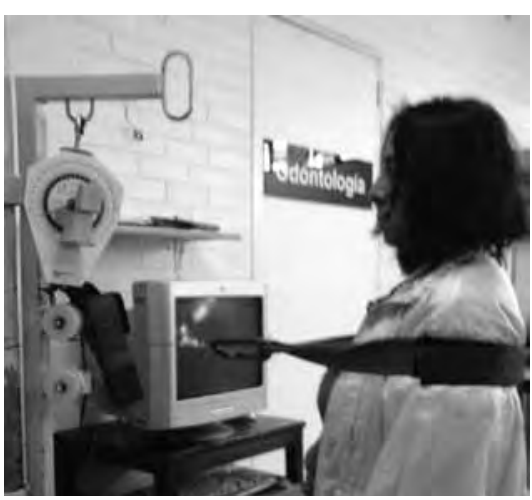

Se efectuaron los cálculos para obtener el índice dinamométrico con la sumatoria de los valores mayores de cada uno de los grupos musculares y se dividió entre el peso de la persona en Kilogramos; siendo la unidad de medida Kilogramos/fuerza (KgFza) (Tabla 1).

\section{Tabla 1. \\ Îndice Dinamométrico.}

\begin{tabular}{ll} 
Mayor de 17 & Excelente \\
14 a 17 & Muy bien \\
\hline 11 a 14 & Bien \\
8 a 11 & Regular \\
\hline Menor de 8 & Mal
\end{tabular}

Fuente: Dr. Miguel Aguilar Casas,

Departamento de Biomecánica, Dirección de Medicina del Deporte, UNAM, Inédito.

La flexoelasticidad se evaluó por segmentos corporales: hombro, cadera, tronco y mano. La flexoelasticidad de hombro, midió el máximo de brazada en hiperextensión lateral y posterior (figura 2).

\section{Figura 2.}

Flexoelasticidad de hombro.

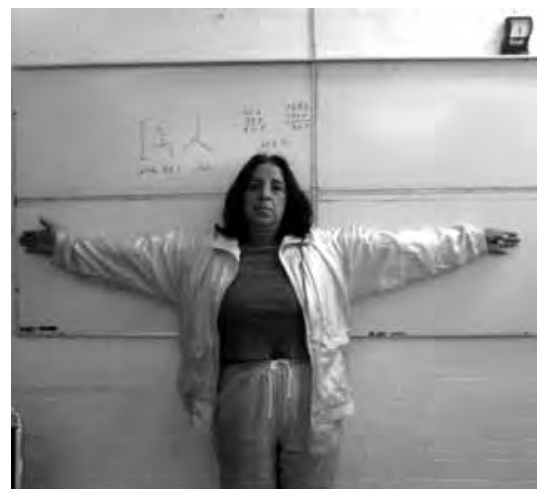

La flexoelasticidad de cadera se midió la talla total, talla sentada y talla descendida (figura 3). Para evaluarla se utilizaron los rangos que se muestran en la tabla 2. 
Figura 3. Talla descendida.

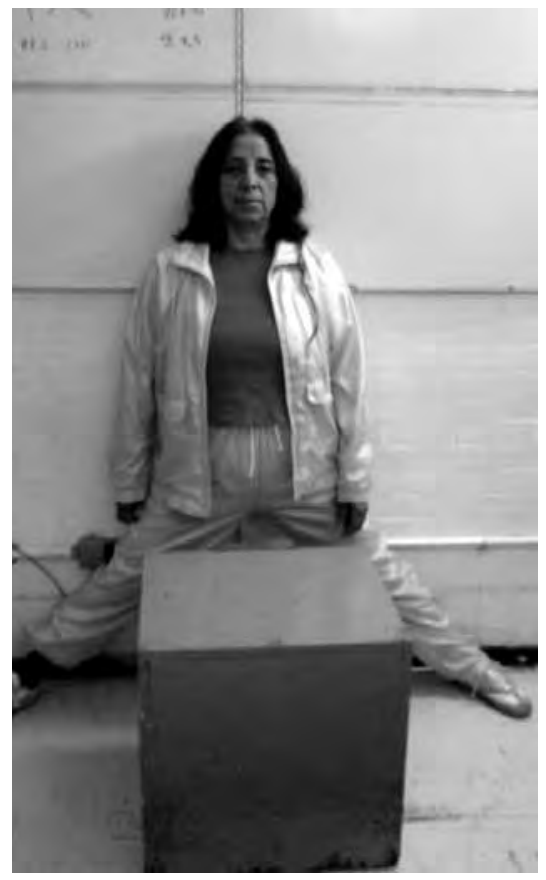

Tabla 2. Calificación

de Flexoelasticidad

hombro y cadera.

\begin{tabular}{ll} 
Mala & $0-20 \%$ \\
Regular & $21-40 \%$ \\
\hline Bueno & $41-60 \%$ \\
Muy Bueno & $61-80 \%$ \\
\hline Excelente & $81-100 \%$
\end{tabular}

Fuente: Dr. Miguel, Aguilar Casas.

Departamento de Biomecánica, Dirección de Medicina del Deporte, UNAM, Inédito.

La flexoelasticidad de tronco se midió con hiperflexión de cadera, miembros pélvicos y torácicos siendo el centro del estadímetro el punto cero con valores negativos hacia arriba y positivos hacia abajo. (Figura 4). Se evaluó de acuerdo a los rangos correspondientes (Tabla 3 ). La flexoelasticidad de mano se calculo a través de hiperflexión de la articulación de la muñeca y el pul-

\section{Figura 4. Prueba de flexoelasticidad de tronco.}

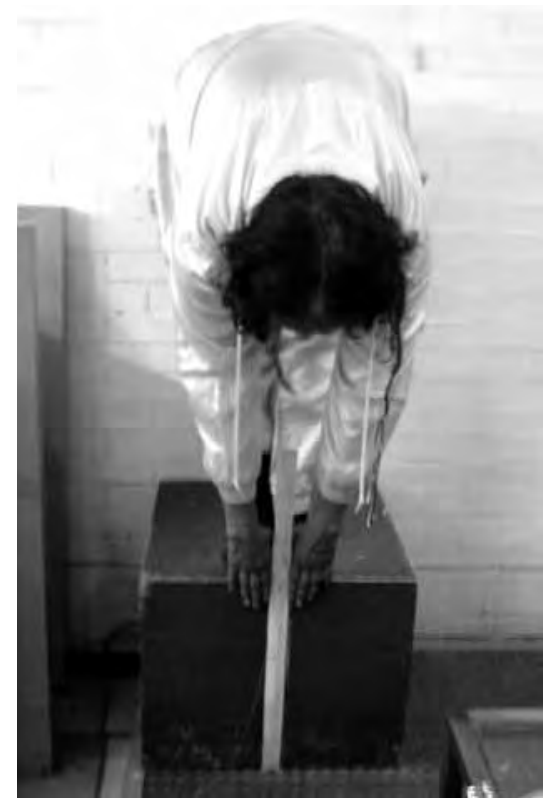

Tabla 3. Calificación de Flexoelasticidad

\section{tronco.}

\section{Mayor}

de $-15 \mathrm{~cm}$

Mal

-15 a $-5 \mathrm{~cm} \quad$ Regular

\begin{tabular}{ll}
\hline$-5 \mathrm{a}+5 \mathrm{~cm}$ & Bien \\
$+5 \mathrm{a}+15 \mathrm{~cm}$ & Muy Bien \\
\hline $\begin{array}{l}\text { Mayor } \\
\text { de }+15 \mathrm{~cm}\end{array}$ & Excelente
\end{tabular}

Fuente: Dr. Miguel, Aguilar Casas.

Departamento de Biomecánica, Dirección

de Medicina del Deporte, UNAM, Inédito.

gar hacia la cara anterior del antebrazo, midiendo la distancia entre la punta del pulgar y el antebrazo, rangos de evaluación (tabla 4.)

Para evaluar la velocidad de reacción, se midió el tiempo entre un estimulo y la integración de una respuesta rápida que amerito una toma de decisión selectiva. Se uti-

\section{Tabla 4. Calificación de Flexoelasticidad de mano.}

\begin{tabular}{ll}
0 a $-1 \mathrm{~cm}$ & Bien \\
$-2 \mathrm{a}-5 \mathrm{~cm}$ & Regular \\
\hline Más & Mal \\
de $-5 \mathrm{~cm}$ &
\end{tabular}

Fuente: Dr. Miguel, Aguilar Casas.

Departamento de Biomecánica, Dirección de Medicina del Deporte, UNAM, Inédito.

lizo el equipo (sistema de análisis biomecánica, SAB4) que consta de 5 plataformas, una central y cuatro distribuidas en forma octagonal. Colocando a la persona en posición ortostática en la plataforma central, se explicó las condiciones de su ejecución: brincar con los pies juntos y lo más rápido posible desplazándose en la dirección en que encendiera la luz (de frente, hacia atrás, a la izquierda y derecha) al finalizar la prueba se calculó el promedio en milésimas de segundo (mseg), ( ).

Para el equilibrio se utilizo el

\section{Tabla 5. Calificación de velocidad \\ de reacción mseg.}

$\begin{array}{ll}\begin{array}{l}\text { Más } \\ \text { de } 1500\end{array} & \text { Malo } \\ 1301 & \text { Regular } \\ \text { a } 1500 & \end{array}$

1101
a 1300

800
a $1100 \quad$ Muy bien

Menos

de 800

Excelente

Fuente: Dr. Miguel, Aguilar Casas.

Departamento de Biomecánica, Dirección de Medicina del Deporte, UNAM, Inédito. 
formato Modified Clinical Test of Sensory Interaction on Balance (M-CTSIB); ${ }^{16}$ se valoró en posición ortostática en superficie estable e inestable (tumbling), con los brazos cruzados a nivel del tórax y con ojos abiertos y cerrados, cada posición en un tiempo de 30 segundos.

El programa de ejerció físico fue de tipo aeróbico, dividido en tres etapas, ver programa de entrenamiento en el cuadro I.

Para el tratamiento estadístico se utilizo el paquete SPSS (V.16) se obtuvieron medidas de tendencia central y dispersión en cada una de las variables: Se utilizo la correlación de Pearson entre la variable independiente ejercicio físico y la dependiente cualidades físicas además de la relación entre características físicas y cualidades físicas.

\section{RESULTADOS}

La muestra se constituyó por 47 mujeres que concluyeron el programa de entrenamiento físico, con promedio de edad $52.6 \pm 4.5$ años (rango 45 a 60 ), talla $1.55 \mathrm{~cm} \pm 5.1$ y peso de $64.4 \mathrm{Kg} \pm 11.2$, las carac- terísticas y cualidades físicas valoradas antes y después del programa de ejercicio físico se muestran en el cuadro II.

Los cambios observados son los siguientes:

Al ingresar al programa solo 4 mujeres tenían una fuerza adecuada es decir muy bien; con el ejercicio fisco se incremento en promedio $1.2 \mathrm{Kg},(\mathrm{r}=.390, \mathrm{p}=.007)$, sin embargo solo 12 mujeres alcanzaron rangos recomendados para esta cualidad (Grafica 1).

\section{Cuadro I. Programa de ejercicio físico.}

\section{Etapa Carga de Trabajo Objetivos}

Primera Duración: 60 min. Frecuencia:

9 semanas

3 días / semana

Intensidad:

$50-60 \%$ de FCMT*
Acondicionamiento físico general con ejercicios básicos de fortalecimiento general y corrección biomecánica del ejercicio.

$\begin{array}{lll} & \text { Duración: } 60 \text { min. Frecuencia: } & \text { - Incremento progresivo de la carga } \\ \text { Segunda } & \text { 3 días /semana } & \text { - Se incluyen ejercicios de fuerza con peso de } 1 \mathrm{~kg} . \\ 12 \text { semanas } & \text { Intensidad: } & \text { - Introducción a los ejercicios de mediano impacto con incremento } \\ & 70-85 \% \text { de FCMT* } & \text { progresivo del estimulo hasta alcanzar el nivel de mantenimiento. }\end{array}$

Duración: 60 min.

Tercera Frecuencia:

10 semanas 3 dias/semana

Intensidad:

- Mantenimiento de un estimulo mínimo de los ejercicios, junto con

$85 \%$ de FCMT* 
Cuadro II. Parámetros obtenidos de las cualidades físicas al inicio y al final del programa de ejercicio físico.

\begin{tabular}{|c|c|c|c|c|}
\hline & \multicolumn{2}{|c|}{$\begin{array}{l}\text { Valoración pre al programa } \\
\text { de ejercicio físico. }\end{array}$} & \multicolumn{2}{|c|}{$\begin{array}{l}\text { Valoración al concluir } \\
\text { el programa de ejercicio físico. }\end{array}$} \\
\hline & $\mathrm{x}$ & D.E. & $\mathrm{x}$ & D. E. \\
\hline Fuerza $(\mathrm{KgFz})$ & 8.54 & 2.068 & 9.82 & 1.89 \\
\hline Velocidad de reacción (mseg) & 1.08 & 1.11 & 1.02 & 1.16 \\
\hline Equilibrio (seg) & 120.98 & 17.85 & 139.91 & 146.96 \\
\hline Flexoelasticidad de mano $(\mathrm{cm})$ & -2.66 & 2.38 & -1.74 & 1.823 \\
\hline $\begin{array}{l}\text { Flexoelasticidad de tronco } \\
(\mathrm{cm})\end{array}$ & .53 & 6.96 & 4.160 & 6.77 \\
\hline Flexoelasticidad de cadera (\%) & 27.91 & 14.87 & 28.64 & 9.190 \\
\hline $\begin{array}{l}\text { Flexoelasticidad de hombro } \\
(\%)\end{array}$ & 86.20 & 12.90 & 87.89 & 14.21 \\
\hline
\end{tabular}

La flexoelasticidad de cadera en 44 mujeres fue regular (Grafica 2), el incremento en promedio fue de un $0.73 \%$.

La flexoelasticidad de mano en la mayoría de mujeres fue regular, ésta se incremento en $0.9 \mathrm{~cm}(\mathrm{r}=$ $.704, \mathrm{p}=.000$ ), la mayoría de mujeres concluyó con una buena flexoelasticidad de mano (Grafica 3).

Ingresaron al programa con una excelente flexoelasticidad de hombro, aun así esta se incremento en $1.6 \%$ aunque estadísticamente no se considera significativo (Grafica 4).

La flexoelasticidad de tronco fue buena (grafica 5), y se incremento en $3.6 \mathrm{~cm},(\mathrm{r}=818, \mathrm{p}=.000)$.

La velocidad de reacción visual se encontró dentro de los rangos recomendados para esta cualidad (grafica 6), incrementando en promedio un $1.1 \mathrm{mseg}(\mathrm{r}=.478, \mathrm{p}=$ $.001)$.

El equilibrio fue deficiente y solo 35 mujeres se encontraba dentro de los rangos recomendados; con el ejercicio físico aumento
Grafica 1. Fuerza de las 47 mujeres antes y al término del programa.

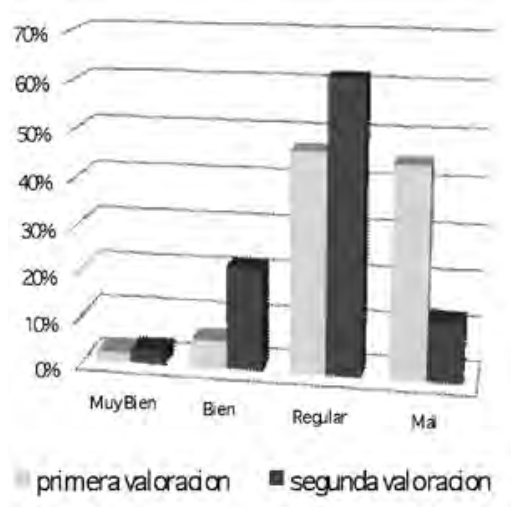

Grafica 3. Flexoelasticidad de mano en la primera y segunda valoración de las 47 mujeres.

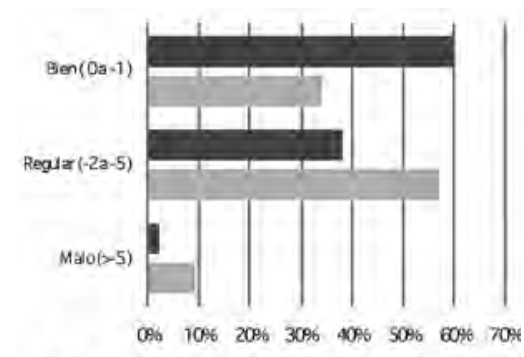

- Seguncavaloradión Aimeravaloradión
Grafica 2. Flexoelasticidad de cadera de las 47 mujeres antes y al término del programa.

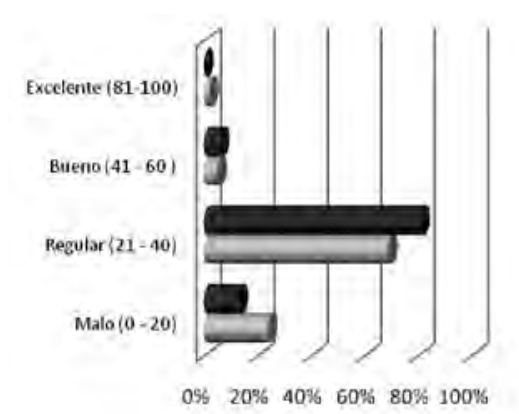

a Segunda valoración EPrimeravaloración
Grafica 4. Flexoelasticidad de hombro de las 47 mujeres al inicio y final del programa.

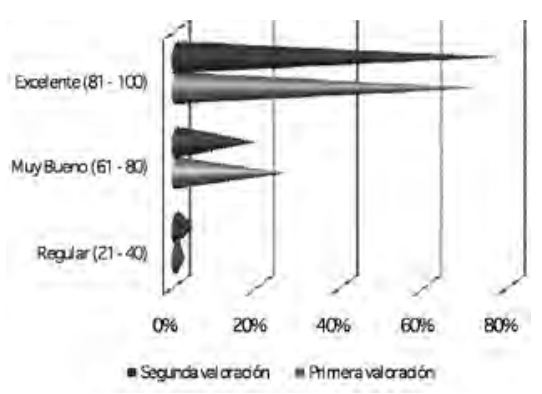


$18.93 \mathrm{seg}$, solo 5 mujeres no mejoraron (grafica 7)

Las cualidades físicas tuvieron asociación con el Índice de Masa Corporal (IMC), Masa magra y masa grasa, la correlación estadísticamente significativa es la siguiente:

Se observó que a mayor IMC $(\mathrm{r}=-.329, \mathrm{p}=.024)$ y masa grasa $(\mathrm{r}=-.382, \mathrm{p}=.008)$ menor fuerza. La flexoelasticidad de cadera disminuye al incrementarse la edad $(\mathrm{r}=-.418, \mathrm{p}=.003)$ y mejora al disminuir la masa grasa $(\mathrm{r}=-.317, \mathrm{p}=$ .030) en esta población.

También existe una asociación entre cualidades físicas: El incremento de la fuerza es directamente proporcional a la flexoelasticidad de cadera $(\mathrm{r}=.312, \mathrm{p}=.033)$ y tronco $(\mathrm{r}=.384, \mathrm{p}=.008)$.

Así mismo la flexoelasticidad de hombro $(\mathrm{r}=.327, \mathrm{p}=.025)$ y tronco $(\mathrm{r}=.313, \mathrm{p}=.032)$ influyen en la de mano.

\section{DISCUSION}

El nivel deficiente de las cualidades físicas observado en la primera valoración de nuestra población de estudio coincide con Carbonell (4) sobre la involución de la fuerza, flexoelasticidad, velocidad de reacción y equilibrio que ocurren durante el transcurso de la vida.

En su conjunto las cualidades físicas al término del programa de ejercicio físico resultaron discretamente beneficiadas, a pesar de que el ejercicio físico no era especifico para mejorarlas, coincidimos con Sánchez Pinilla ${ }^{10}$ en lo referente a que la capacidad para mejorarlas dependerá de la cantidad de trabajo y tipo de ejercicio.

Se Corrobora que las cualidades físicas se pueden mejorar en adultas

\section{Grafica 5. Flexoelasticidad de tronco de las 47 mujeres antes y al término del programa.}

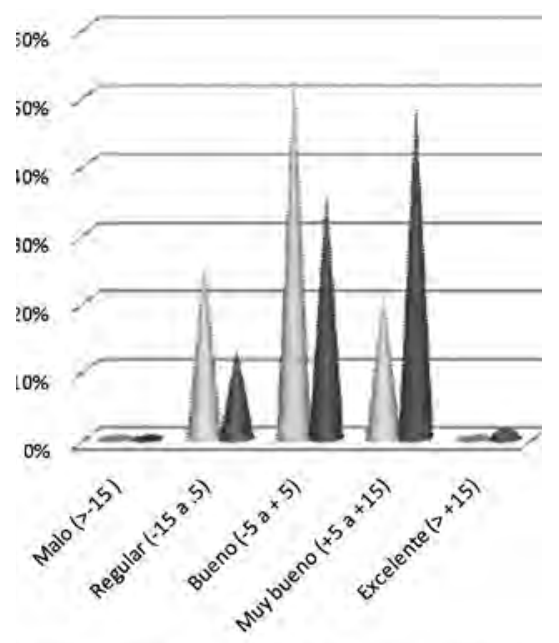

primeravaloración Segunda valoraciı

\section{Grafica 7. Equilibrio de las 47 mujeres antes y al término del programa.}

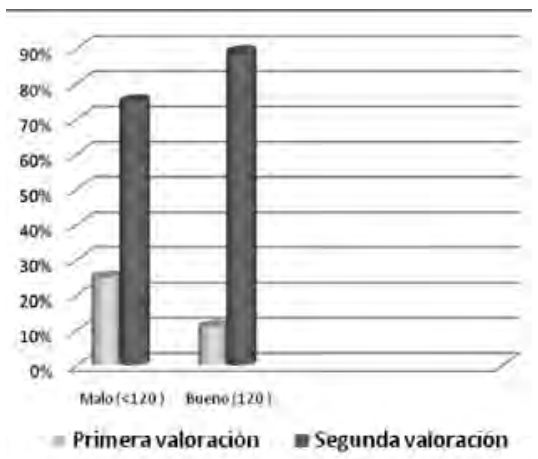

mayores sedentarias como fue en el caso del presente estudio. Las cualidades físicas con mayor incremento fueron la fuerza y la flexoelasticidad.

\section{CONCLUSION}

Este método de estudio proporciona una herramienta que puede ser empleada para analizar los efectos del ejercicio físico sobre las cualidades físicas, sin embargo, se requiere de un programa de entrenamiento de mayor tiempo y sin interrupciones, debido a que a causas externas al programa (Influenza y vacaciones administrativas de la UNAM) se interrumpió con lapsos de tiempo que impactaron en los resultados del acondicionamiento físico para perder los efectos positivos del entrenamiento físico sobre las cualidades.

Por último se considera necesario el continuar con este tipo de estudios sobre las cualidades físicas en población mexicana en proceso de envejecimiento que nos permitan definir y dar a conocer la dosificación del ejercicio para mejorar las cualidades físicas y con ello la calidad de vida. 


\section{REFERENCIAS BIBLIOGRÁFICAS}

1. Ham C. "Estudio demográficos y urbanos: Esperanzas de vida y expectativas de salud en las edades avanzadas" 2001 (consultado 31/01/2010 on line). Disponible en: www.colmex.mx/centros/ ceddu/revista/rev48.htm.

2. Cabrera RA, Comportamiento frente a la práctica de actividades físicodeportivas de tiempo libre y su relación entre algunas variables sociodemográficas de los adultos mayores de 60 años del municipio de Tapachula, Chiapas, México. Revista mexicana de investigación en cultura física y deporte.2009; 1(1):54-77

3. Gonzáles CA, Becerra PA, Carmona SF, Cerezo GI, Hernández HH, Lara EA. Ejercicio físico para la salud. Rev Mex Cardiol 2001; 12(4): 168-180.

4. Carbonell B. "Involución de la condición física por el envejecimiento: Fuerza de tren superior, fuerza del tren inferior, flexibilidad y equilibrio" 2009 (consultado 24/01/2010 on line). Disponible en: www. apunts.org/apunts/ctl.servlet?_f $=40$.

5. Bueno A, Valencia G, Noguez M. "Crece en México la población de viejos: En México, siete millo- nes de personas son mayores de 60 años". (Consultado 31-01-2010 on line). Disponible en: www.elsiglodetorreon.com.mx/noticia/439. crece-en-mexico-la-poblacion-deviejos.html.

6. Fuentes M.l. "Adultos mayores riesgo y pobreza: México en ruta de envejecimiento" 2010 (consultado 13/09/2010 on line). Disponible en: www.correo-gto.com.mx/notas.asp?id=181032.

7. INEGI. "Dinámica poblacional nacional” 2010 (consultado 13/09/2010 on line). Disponible en: www. inegi.org.mx/inegi/default. aspx?s=estc $=124$.

8. Dietrich M, Klaus C, Klaus L. Manual de Metodología del Entrenamiento Deportivo. $1^{\text {a }}$ Ed. Paidotribo. Barcelona, 2001. 70-78 pp.

9. Levesque D. Entrenamiento en los Deportes. $1^{\text {a }}$ Ed. Paidotribo. Barcelona, 1893. 43-52 pp.

10. Sánchez - Pinilla R. Medicina del Ejercicio Físico y del Deporte para la

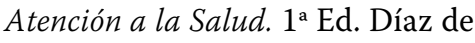
Santos. Madrid, 1992. 42- 97 pp.

11. Acosta CB, Aranda AJ, Reyes MH. Patrones de actividad física de la mujer y del hombre. Rev. Med Inst Mex Seguro Soc 2006; 44 (supl1): S79 - S86.
12. Mora J. Teoría del Entrenamiento y del Acondicionamiento Deportivo. $1^{a}$ Ed. COplef. Andalucía - Córdoba, 1995. 398-401 pp.

13. Suárez H Arocena M. "Las alteraciones del equilibrio en el Adulto Mayor: Alteraciones en el procesamiento de la información sensorial" 2009 (consultado 15/02/2010 on line). Disponible en: www.clinicalascondes.cl/area_academica/ pdf/MED20_4/401_ALTERACIONES_EQUILIBRIO.PDF.REV.MED:C UN:CONDES2009;20(4)401-407.

14. Wales J. "Entrenamiento: Entrenamiento deportivo" 2010 (consultado 13/09/2010 on line). Disponible en: http://es.wikipedia.org/wiki/ Entrenamiento.

15. Wales J. "Aprendizaje: Definición de aprendizaje" 2010 (consultado 13/09/2010 on line). Disponible en: http://es.wikipedia.org/wiki/ Aprendizaje.

16. Debra J. Rose, Allison L. "Sensory Reception and organization: Modified clinical test of sensory interaction on balance (mCTSIB)"2009 (consultado 15/02/2010 on line). Disponible en: http://resourcesonbalance.com/neurocom/protocols/sensoryImpairments/mCTsib.aspx 